Supplementary Information for

\title{
Circularly Polarized Luminescence from Chiral Tetranuclear Copper(I) Iodide Clusters
}

Li Yao ${ }^{1}$, Guangda Niu ${ }^{1}$, Junze $\mathrm{Li}^{2}$, Liang $\mathrm{Gao}^{1}$, Xufeng $\mathrm{Luo}^{3}$, Bing Xia ${ }^{1}$, Yuhao $\mathrm{Liu}^{1}$, Peipei Du ${ }^{1}$, Dehui $\mathrm{Li}^{2}$, Chao Chen ${ }^{1}$, Youxuan Zheng ${ }^{3}$, Zewen Xiao ${ }^{1}$, Jiang Tang ${ }^{1,2 *}$

${ }^{1}$ Wuhan National Laboratory for Optoelectronics, Huazhong University of Science and Technology, Wuhan 430074, China

${ }^{2}$ School of Optical and Electronic Information, Huazhong University of Science and Technology, Wuhan 430074, China

${ }^{3}$ State Key Laboratory of Coordination Chemistry, School of Chemistry and Chemical Engineering, Nanjing University, Nanjing 210023, China

E-mail: guangda_niu@hust.edu.cn; jtang@mail.hust.edu.cn 


\section{Table of Contents}

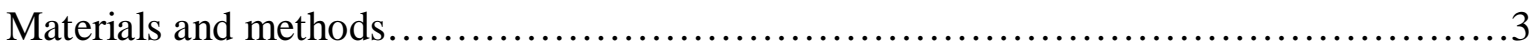

Equation S1. The derivation process for figure of merit: $\mathrm{FM}=g_{\mathrm{lum}} \times \mathrm{PLQY} \ldots \ldots \ldots \ldots \ldots \ldots \ldots . . . \ldots$

Table S1. The selected parameters for various good CPL materials..........................7

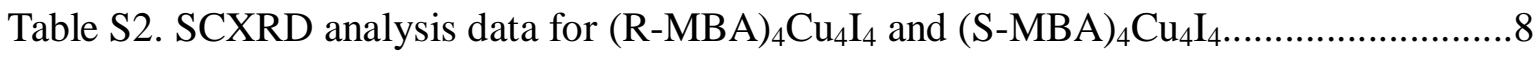

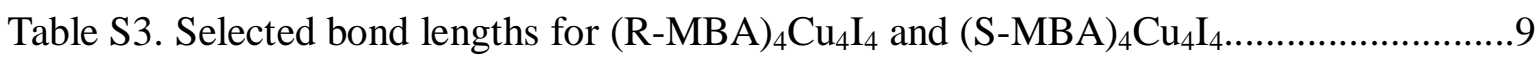

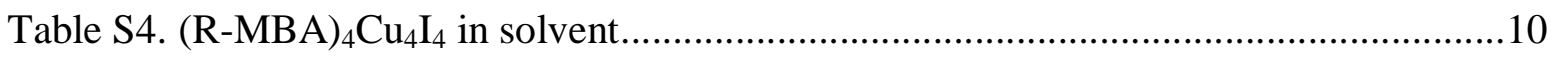

Figure S1. Crystalline structure of (R-MBA) $)_{4} \mathrm{Cu}_{4} \mathrm{I}_{4}$ and (S-MBA) $)_{4} \mathrm{Cu}_{4} \mathrm{I}_{4} \ldots \ldots \ldots \ldots \ldots \ldots \ldots \ldots \ldots \ldots . . . \ldots 11$

Figure S2. XRD patterns of (R-MBA) ${ }_{4} \mathrm{Cu}_{4} \mathrm{I}_{4}$ and (S-MBA) ${ }_{4} \mathrm{Cu}_{4} \mathrm{I}_{4}$ powders..........................11

Figure S3. PL and PLE spectra of (R-MBA) ${ }_{4} \mathrm{Cu}_{4} \mathrm{I}_{4}$ crystals..............................................12

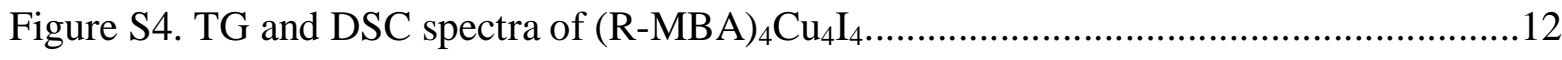

Figure S5. PL stability of (R-MBA) $)_{4} \mathrm{Cu}_{4} \mathrm{I}_{4}$ and $(\mathrm{S}-\mathrm{MBA})_{4} \mathrm{Cu}_{4} \mathrm{I}_{4}$ in water..................13

Figure S6. PLQY spectra of (R-MBA) ${ }_{4} \mathrm{Cu}_{4} \mathrm{I}_{4}$ and (S-MBA) $)_{4} \mathrm{Cu}_{4} \mathrm{I}_{4}$ clusters..........................13

Figure S7. Photographs of pressed (R-MBA) ${ }_{4} \mathrm{Cu}_{4} \mathrm{I}_{4}$ and (S-MBA) ${ }_{4} \mathrm{Cu}_{4} \mathrm{I}_{4}$ pellets...................14

Figure S8. Absorbance and the corresponding CD spectra for the R-MBA and S-MBA.......14

Figure S9. Calculated absorption and oscillator strength spectra of (R-MBA) ${ }_{4} \mathrm{Cu}_{4} \mathrm{I}_{4} \ldots \ldots \ldots .15$

Figure S10. Calculated hole-electron distributions for selected excited states................16

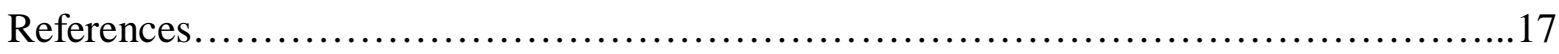




\section{Materials and methods}

Materials. R- and S-methylbenzylamine ( $\geq 99 \%$ ) were purchased from Alfa Aesar. Ethanol, hexane and copper(I) iodide (CuI, $\geq 99 \%$ ) were bought from Sinopharm Chemical Reagent Co., Ltd., China. All materials and reagents were used as received.

Cluster synthesis. The clusters were synthesized through mixing CuI powder (10 mmol, 1.90 g) and R/S-MBA (10 mmol, $1.30 \mathrm{~mL})$ into ethanol (225 mL). After stirring for 5 minutes, the precipitation was filtrated and washed three times with hexane, then dried under vacuum (at room temperature) for $3 \mathrm{~h}$. This process resulted in a product yield of $80 \%$. The single crystals were grown through leaving the precursor solution overnight at room temperature, and then the crystals were washed with hexane and dried naturally in the air.

Characterization Methods. Single crystal X-ray diffraction (SCXRD) data of the single crystals was collected at room temperature using an XtaLAB PRO MM007HF diffractometer with $\mathrm{Cu}$ Ka radiation. Powder XRD were measured by Philips X'pert pro-MRD diffractometer with $\mathrm{Cu} \mathrm{K} \alpha$ radiation.

A UV-vis spectrophotometer (PerkinElmer Instruments, Lambda 950 using integrating sphere) was used to measure the absorption spectra. PL and PLE measurements were carried out using Edinburgh FLS920. The time-resolved PL was recorded on a time-correlated single photon-counting system (TCSPC, PicoQuant GmbH) with a microsecond Xenon lamp as the excitation source.

An absolute photoluminescence measurement system (Hamamatsu Quantaurus-QY) was used to measure PLQY. The excitation wavelength was $360 \mathrm{~nm}$, and the step increments and integration time were set as $1 \mathrm{~nm}$ and $0.5 \mathrm{~s}$, respectively. Commercial YAG: $\mathrm{Ce}^{3+}$ powder from Hunan LED Company with a standard PLQY of 80\%-85\% (460 nm excitation) was used to calibrate the system. 
Transmission CD spectra were collected using a CD spectrometer (J810, JASCO) with “Standard” sensitivity (100 mdeg) at $100 \mathrm{~nm} \min ^{-1}$ scan speed. The samples for CD measurement were prepared by uniformly grinding the crystals with $\mathrm{KBr}$ into fine powder and then pressing into pellets. ${ }^{1}$ CPL spectra were recorded by a JASCO CPL-300 instrument in the solid state with scanning speed of $200 \mathrm{~nm} / \mathrm{min}$. The ground solid-state samples (5 mg) were uniformly sandwiched by two quartz slides. The excitation wavelength was $360 \mathrm{~nm}$. Each sample was measured for at least three times to confirm the reproducibility.

Theoretical simulation. Density-functional theory (DFT) calculations based on crystal cell structure were performed using the projection-augmented wave (PAW) method as implemented in the Vienna Ab initio Simulation Package (VASP) 5.4 .4 code. $^{2}$ The plane-wave cutoff energy was set to $500 \mathrm{eV}$. The generalized gradient approximation (GGA) Perdew-Burke-Ernzerhof (PBE) ${ }^{3}$ was employed as the exchange-correlation functional. The Brillouin zone was sampled using a $3 \times 2 \times 1$ MonkhorstPack (MP) grid. ${ }^{4}$ Prior to the band structure and density of state calculations, the crystal structure was fully relaxed until the total force on each atom was $<0.01 \mathrm{eV} / \AA$.

The DFT and time-dependent DFT (TD-DFT) calculations were performed using the Gaussian16 software. ${ }^{5}$ Based on the structures obtained in experiments, geometry optimization were applied by optimizing $\mathrm{H}$ atoms and freezing $\mathrm{Cu}, \mathrm{I}, \mathrm{C}, \mathrm{N}$ atoms, using the PBE1PBE (PBE0) ${ }^{3,} 6$ function and the def2-tzvp base group. The TD-DFT calculation was performed at PBE1PBE/def2-svp level, based on the optimized ground state structure. In the calculation of UV-vis transitions and electrical CD, 500 singlet to singlet transitions were computed. The calculated UV-VIS and ECD spectra were shifted $1.1 \mathrm{eV}$ in energy in order to match the experimental results. The hole-electron compositions were obtained by the Hirshfeld method ${ }^{7}$ and Multiwfn packages. ${ }^{8}$ 
Equation S1. The derivation process for figure of merit: $F M=g_{\text {lum }} \times \mathrm{PLQY}$

The detailed deviation process for figure of merit $(\mathrm{FM})$, here $\mathrm{FM}=g_{\mathrm{lum}} \times \mathrm{PLQY}==2 \frac{N_{L}-N_{R}}{N_{a}}$

(Equation S1), when the $g_{\lambda}$ ( $g_{\text {lum }}$ at the wavelength of $\lambda$ ) value stay the same in the whole luminescence spectra.

$$
\begin{gathered}
g_{\lambda}=2 \frac{I_{L \lambda}-I_{R \lambda}}{I_{L \lambda}+I_{R \lambda}}=2 \frac{N_{L \lambda} h v_{\lambda}-N_{R \lambda} h v_{\lambda}}{N_{L \lambda} h v_{\lambda}+N_{R \lambda} h v_{\lambda}}=2 \frac{N_{L \lambda}-N_{R \lambda}}{N_{L \lambda}+N_{R \lambda}} \\
N_{e}=\int\left(N_{L \lambda}+N_{R \lambda}\right) d \lambda=\sum_{\lambda}\left(N_{L \lambda}+N_{R \lambda}\right)=\sum_{n}\left(N_{L n}+N_{R n}\right) \\
=N_{L 1}+N_{R 1}+N_{L 2}+N_{R 2}+N_{L 3}+N_{R 3} \cdots \\
g_{\lambda 1}=g_{\lambda 2}=g_{\lambda 3}=2 \frac{N_{L 1}-N_{R 1}}{N_{L 1}+N_{R 1}}=2 \frac{N_{L 2}-N_{R 2}}{N_{L 2}+N_{R 2}}=2 \frac{N_{L 3}-N_{R 3}}{N_{L 3}+N_{R 3}} \\
P L Q Y=\frac{N_{e}}{N_{a}}
\end{gathered}
$$

Here, $N_{e}$ represent total number of emitted photons, $N_{a}$ represent total number of absorbed photons, $N_{L}$ represent total number of left-handed photons emitted, $N_{R}$ represent total number of right-handed photons emitted, $N_{L 1}$ and $N_{R 1}$ represent the number of left-handed photons and right-handed photons emitted at the wavelength of $\lambda 1$.

From equation (1)-(4), we can get:

$$
\begin{aligned}
& g_{\lambda} \times P L Q Y=g_{\lambda 1} \times P L Q Y=2 \frac{N_{L 1}-N_{R 1}}{N_{L 1}+N_{R 1}} \times \frac{N_{e}}{N_{a}} \\
& =2 \frac{N_{L 1}-N_{R 1}}{N_{L 1}+N_{R 1}} \times \frac{\left(N_{L 1}+N_{R 1}+N_{L 2}+N_{R 2}+N_{L 3}+N_{R 3} \cdots\right)}{N_{a}} \\
& =2 \frac{N_{L 1}-N_{R 1}}{N_{L 1}+N_{R 1}} \times \frac{\left(N_{L 1}+N_{R 1}\right)}{N_{a}}+2 \frac{N_{L 1}-N_{R 1}}{N_{L 1}+N_{R 1}} \times \frac{\left(N_{L 2}+N_{R 2}\right)}{N_{a}}+2 \frac{N_{L 1}-N_{R 1}}{N_{L 1}+N_{R 1}} \times \frac{\left(N_{L 3}+N_{R 3}\right)}{N_{a}}+\cdots \\
& =2 \frac{N_{L 1}-N_{R 1}}{N_{L 1}+N_{R 1}} \times \frac{\left(N_{L 1}+N_{R 1}\right)}{N_{a}}+2 \frac{N_{L 2}-N_{R 2}}{N_{L 2}+N_{R 2}} \times \frac{\left(N_{L 2}+N_{R 2}\right)}{N_{a}}+2 \frac{N_{L 3}-N_{R 3}}{N_{L 3}+N_{R 3}} \times \frac{\left(N_{L 3}+N_{R 3}\right)}{N_{a}}+\cdots
\end{aligned}
$$


$=2 \frac{\left(N_{L 1}-N_{R 1}\right)}{N_{a}}+2 \frac{\left(N_{L 2}-N_{R 2}\right)}{N_{a}}+2 \frac{\left(N_{L 3}-N_{R 3}\right)}{N_{a}}+\cdots$
$=2 \frac{\left(N_{L 1}+N_{L 2}+N_{L 3}+\cdots\right)-\left(N_{R 1}+N_{R 2}+N_{R 3}+\cdots\right)}{N_{a}}$

$=2 \frac{N_{L}-N_{R}}{N_{a}}$

It follows that $g_{\text {lum }} \times \mathrm{PLQY}=2 \frac{N_{L}-N_{R}}{N_{a}}= \pm 2$ means the absorbed photons completely transformed into left-handed photons or right-handed photons. 
Table S1 The compiled $g_{\text {lum }}$ and PLQY values for various good CPL materials reported in the literature.

\begin{tabular}{|c|c|c|c|c|c|c|}
\hline $\begin{array}{l}\text { Materials } \\
\text { Categories }\end{array}$ & Examples & $\begin{array}{l}\text { PLQY } \\
(\%) \\
\end{array}$ & $g_{\text {lum }}$ & $\begin{array}{l}\text { FM } \\
\left(q_{\text {lum }} \times \mathrm{PLQY}\right)\end{array}$ & State & $\begin{array}{l}\text { Referenc } \\
\text { es }\end{array}$ \\
\hline \multirow{6}{*}{$\begin{array}{l}\text { Lanthanide } \\
\text { complexes }\end{array}$} & $\mathrm{Cs}[\mathrm{Eu}((+)-\mathrm{hfbc}) 4]$ & $3^{\text {a) }}$ & +1.41 & / & In $\mathrm{CHCl}_{3}$ & 9 \\
\hline & $\mathrm{Cs}[\operatorname{Eu}((-)-\mathrm{hfbc}) 4]$ & & -1.41 & / & & \\
\hline & Trinuclear & 25 & +0.45 & / & In acetonitrile & 10 \\
\hline & $\Delta \Delta \Delta-\left[\mathrm{Eu}(\mathrm{S}-\mathrm{Phbipox})_{2}\right]_{3}^{3+}$ & & & & & \\
\hline & [TbR(+)BnMeH22IAM] & 63 & +0.044 & / & In methanol & 11 \\
\hline & [TbS(-)BnMeH22IAM] & & -0.048 & / & & \\
\hline \multirow{5}{*}{$\begin{array}{l}\text { Small } \\
\text { molecular }\end{array}$} & (S)-1 & 88 & +0.003 & +0.0026 & In toluene & 12 \\
\hline & (R)-1 & & -0.003 & -0.0026 & & \\
\hline & $\begin{array}{l}\text { Helicene-like } \\
\text { (M)-6 }\end{array}$ & 30 & -0.032 & -0.0096 & In $\mathrm{CHCl}_{3}$ & 13 \\
\hline & Naphthodioxepin & 90 & +0.0022 & +0.0020 & In 1,4-dioxane & 14 \\
\hline & octamer & 22 & +0.007 & +0.0015 & Solid State & \\
\hline polymer & $\begin{array}{l}\text { Chiral } \beta \text {-Ketoiminate- } \\
\text { Based polymer (P-1) }\end{array}$ & 37 & +0.349 & +0.13 & In $\mathrm{CH}_{2} \mathrm{Cl}_{2}$ & 15 \\
\hline Transition-meta & $\Lambda$-Ir(ррру) ${ }_{3}$ & 64 & +0.0028 & +0.0018 & In acetonitrile & 16 \\
\hline \multirow[t]{9}{*}{1 complexes } & $\Delta$-Ir(ррру) $)_{3}$ & & -0.0032 & -0.0020 & & \\
\hline & Chiral Zn complexes & 55 & -0.001 & -0.00055 & In $\mathrm{CH}_{2} \mathrm{Cl}_{2}$ & 17 \\
\hline & & 59 & +0.001 & +0.00059 & & \\
\hline & Chial gold cluser-based & / & -0.007 & / & In $70 \%$ n-hexane & 18 \\
\hline & crystalline nanocubes & l & +0.007 & / & & \\
\hline & $\mathrm{D} / \mathrm{L}-\mathrm{Cu}_{5 \mathrm{~V}}$ & 47. & +0.0098 & +0.0046 & Solid state & 19 \\
\hline & & 47 & -0.0098 & -0.0046 & & \\
\hline & $(\mathrm{R}-\mathrm{MBA})_{4} \mathrm{Cu}_{4} \mathrm{I}_{4}$ & 53 & -0.008 & -0.00424 & Powder & This \\
\hline & $(\mathrm{S}-\mathrm{MBA}){ }_{4} \mathrm{Cu}_{4} \mathrm{I}_{4}$ & 60 & +0.01 & +0.006 & & work \\
\hline \multirow[t]{10}{*}{ Perovskite } & R-RDCP & 90 & $+0.06(2 \mathrm{~K})$ & +0.054 & Film & 20 \\
\hline & S-RDCP & & $-0.06(2 \mathrm{~K})$ & -0.054 & & \\
\hline & $(\mathrm{S}-\mathrm{MBA})_{2} \mathrm{PbI}_{4}$ & / & $+0.192(77 \mathrm{~K})$ & / & Single crystal & 21 \\
\hline & $(\mathrm{R}-\mathrm{MBA})_{2} \mathrm{PbI}_{4}$ & I & $-0.202(77 K)$ & l & & \\
\hline & $(\mathrm{S})-\alpha-(\mathrm{PEA})_{2} \mathrm{PbI}_{4}$ & / & 0.228 & / & Single crystal & 22 \\
\hline & $(\mathrm{R})-\alpha-(\mathrm{PEA})_{2} \mathrm{PbI}_{4}$ & / & 0.274 & / & & \\
\hline & $\mathrm{C}_{4} \mathrm{~N}_{2} \mathrm{H}_{14} \mathrm{PbI}_{4}$ & / & $0.076(77 \mathrm{~K})$ & / & Single crystal & 22 \\
\hline & R-Pero-NCs & l & 0.0035 & / & In n-hexane & 23 \\
\hline & S-Pero-NCs & l & -0.0023 & / & & \\
\hline & $\mathrm{CsPbBr} 3 \mathrm{NCs}$ & 1 & 0.007 & / & In chiral DGAm gel & 24 \\
\hline
\end{tabular}

Note: a) The results were derived in solid state; The $(2 \mathrm{~K})$ in the glum column stands for the temperature, at which temperature the glum value is measured. Unlabeled is measured at room temperature; / means not available. 
Table S2 Single cystal X-ray diffraction analysis data and structure refinement at room temperature for (R-MBA) ${ }_{4} \mathrm{Cu}_{4} \mathrm{I}_{4}$ and (S-MBA) ${ }_{4} \mathrm{Cu}_{4} \mathrm{I}_{4}$.

\begin{tabular}{|c|c|c|}
\hline Compounds & $(\mathrm{R}-\mathrm{MBA})_{4} \mathrm{Cu}_{4} \mathrm{I}_{4}$ & $(\mathrm{~S}-\mathrm{MBA})_{4} \mathrm{Cu}_{4} \mathrm{I}_{4}$ \\
\hline Chemical formula & $\mathrm{C}_{32} \mathrm{H}_{44} \mathrm{Cu}_{4} \mathrm{I}_{4} \mathrm{~N}_{4}$ & $\mathrm{C}_{32} \mathrm{H}_{44} \mathrm{Cu}_{4} \mathrm{I}_{4} \mathrm{~N}_{4}$ \\
\hline Formula weight & 1246.47 & 1246.47 \\
\hline Temperature/k & 295.65(10) & 294.7 \\
\hline Crystal system & orthorhombic & orthorhombic \\
\hline Space group & $P 2_{1} 2_{1} 2_{1}$ & $P 2_{1} 2_{1} 2_{1}$ \\
\hline$a(\AA)$ & $7.54153(19)$ & $7.54722(4)$ \\
\hline$b(\AA)$ & $12.0777(4)$ & $12.06809(5)$ \\
\hline$c(\AA)$ & 45.1161(16) & $45.0822(2)$ \\
\hline$\alpha\left({ }^{\circ}\right)$ & 90 & 90 \\
\hline$\beta\left(^{\circ}\right)$ & 90 & 90 \\
\hline$\gamma\left({ }^{\circ}\right)$ & 90 & 90 \\
\hline Volume $\left(\AA^{3}\right)$ & $4109.4(2)$ & $4106.11(3)$ \\
\hline Z & 4 & 4 \\
\hline$\rho_{\text {calc }}\left(\mathrm{g} \mathrm{cm}^{-3}\right)$ & 2.015 & 2.016 \\
\hline$\mu\left(\mathrm{mm}^{-1}\right)$ & 26.114 & 26.135 \\
\hline $\mathrm{F}(000)$ & 2368.0 & 2368.0 \\
\hline $2 \theta$ range for data collection $\left(^{\circ}\right)$ & 7.578 to 147.678 & 7.584 to 147.89 \\
\hline Reflections collected & 20862 & 103927 \\
\hline Independent reflections & $8036\left[R_{\mathrm{int}}=0.0512, R_{\mathrm{sigma}}=0.0571\right]$ & $\begin{array}{l}8200\left[R_{\text {int }}=0.0662, R_{\text {sigma }}=\right. \\
0.0209]\end{array}$ \\
\hline Data/restraints/parameters & $8036 / 0 / 401$ & $8200 / 0 / 401$ \\
\hline Goodness-of-fit on $F^{2}$ & 1.016 & 1.095 \\
\hline Final $R$ indexes $[\mathrm{I}>=2 \sigma(I)]$ & $R_{1}=0.0418, w R_{2}=0.0992$ & $R_{1}=0.0295, w R_{2}=0.0738$ \\
\hline Final $R$ indexes [all data] & $R_{1}=0.0551, w R_{2}=0.1080$ & $R_{1}=0.0298, w R_{2}=0.0740$ \\
\hline Largest diff. peak/hole (e $\AA^{-3}$ ) & $0.70 /-0.86$ & $0.48 /-1.25$ \\
\hline Flack parameter & $-0.005(5)$ & $0.000(2)$ \\
\hline
\end{tabular}


Table S3. Selected bond lengths $(\AA)$ for (R-MBA) ${ }_{4} \mathrm{Cu}_{4} \mathrm{I}_{4}$ and (S-MBA) ${ }_{4} \mathrm{Cu}_{4} \mathrm{I}_{4}$.

\begin{tabular}{|c|c|c|c|c|c|}
\hline \multicolumn{3}{|c|}{$(\mathrm{S}-\mathrm{MBA})_{4} \mathrm{Cu}_{4} \mathrm{I}_{4}$} & \multicolumn{3}{|c|}{$(\mathrm{R}-\mathrm{MBA})_{4} \mathrm{Cu}_{4} \mathrm{I}_{4}$} \\
\hline Atom & Atom & Length/Å & Atom & Atom & Length/ $\AA$ \\
\hline $\mathrm{I}(1)$ & $\mathrm{Cu}(1)$ & $2.7636(13)$ & $\mathrm{I}(1)$ & $\mathrm{Cu}(1)$ & $2.762(2)$ \\
\hline $\mathrm{I}(1)$ & $\mathrm{Cu}(3)$ & $2.7701(13)$ & $\mathrm{I}(1)$ & $\mathrm{Cu}(3)$ & $2.766(2)$ \\
\hline $\mathrm{I}(1)$ & $\mathrm{Cu}(4)$ & $2.6173(12)$ & $\mathrm{I}(1)$ & $\mathrm{Cu}(4)$ & $2.619(2)$ \\
\hline $\mathrm{I}(2)$ & $\mathrm{Cu}(1)$ & 2.7543(13) & $\mathrm{I}(2)$ & $\mathrm{Cu}(2)$ & $2.761(2)$ \\
\hline $\mathrm{I}(2)$ & $\mathrm{Cu}(2)$ & $2.6862(12)$ & $\mathrm{I}(2)$ & $\mathrm{Cu}(3)$ & $2.734(2)$ \\
\hline $\mathrm{I}(2)$ & $\mathrm{Cu}(3)$ & $2.6704(12)$ & $\mathrm{I}(2)$ & $\mathrm{Cu}(4)$ & $2.606(2)$ \\
\hline $\mathrm{I}(3)$ & $\mathrm{Cu}(2)$ & $2.7610(12)$ & $\mathrm{I}(3)$ & $\mathrm{Cu}(2)$ & $2.686(2)$ \\
\hline I(3) & $\mathrm{Cu}(3)$ & $2.7337(12)$ & $\mathrm{I}(3)$ & $\mathrm{Cu}(1)$ & $2.754(2)$ \\
\hline $\mathrm{I}(3)$ & $\mathrm{Cu}(4)$ & 2.6087(13) & $\mathrm{I}(3)$ & $\mathrm{Cu}(3)$ & $2.674(2)$ \\
\hline $\mathrm{I}(4)$ & $\mathrm{Cu}(1)$ & $2.6323(12)$ & $\mathrm{I}(4)$ & $\mathrm{Cu}(2)$ & $2.678(2)$ \\
\hline $\mathrm{I}(4)$ & $\mathrm{Cu}(2)$ & $2.6820(13)$ & $\mathrm{I}(4)$ & $\mathrm{Cu}(1)$ & $2.632(2)$ \\
\hline $\mathrm{I}(4)$ & $\mathrm{Cu}(4)$ & 2.9731(15) & $\mathrm{I}(4)$ & $\mathrm{Cu}(4)$ & $2.967(3)$ \\
\hline $\mathrm{Cu}(1)$ & $\mathrm{Cu}(2)$ & $2.6419(15)$ & $\mathrm{Cu}(2)$ & $\mathrm{Cu}(1)$ & $2.644(3)$ \\
\hline $\mathrm{Cu}(1)$ & $\mathrm{Cu}(3)$ & $2.6384(16)$ & $\mathrm{Cu}(2)$ & $\mathrm{Cu}(3)$ & $2.739(3)$ \\
\hline $\mathrm{Cu}(1)$ & $\mathrm{Cu}(4)$ & $2.7325(15)$ & $\mathrm{Cu}(2)$ & $\mathrm{Cu}(4)$ & $2.629(3)$ \\
\hline $\mathrm{Cu}(1)$ & $\mathrm{N}(1)$ & $2.060(5)$ & $\mathrm{Cu}(2)$ & $\mathrm{N}(1)$ & $2.055(10)$ \\
\hline $\mathrm{Cu}(2)$ & $\mathrm{Cu}(3)$ & $2.7307(14)$ & $\mathrm{Cu}(1)$ & $\mathrm{Cu}(3)$ & $2.642(3)$ \\
\hline $\mathrm{Cu}(2)$ & $\mathrm{Cu}(4)$ & 2.6301(16) & $\mathrm{Cu}(1)$ & $\mathrm{Cu}(4)$ & $2.732(3)$ \\
\hline $\mathrm{Cu}(2)$ & $\mathrm{N}(2)$ & $2.056(5)$ & $\mathrm{Cu}(1)$ & $\mathrm{N}(2)$ & $2.055(9)$ \\
\hline $\mathrm{Cu}(3)$ & $\mathrm{Cu}(4)$ & 2.6798(16) & $\mathrm{Cu}(3)$ & $\mathrm{Cu}(4)$ & $2.683(3)$ \\
\hline $\mathrm{Cu}(3)$ & $\mathrm{N}(3)$ & $2.054(5)$ & $\mathrm{Cu}(3)$ & $\mathrm{N}(3)$ & $2.055(9)$ \\
\hline $\mathrm{Cu}(4)$ & $\mathrm{N}(4)$ & $2.037(6)$ & $\mathrm{Cu}(4)$ & $\mathrm{N}(4)$ & $2.043(10)$ \\
\hline
\end{tabular}


Table S4. (R-MBA) ${ }_{4} \mathrm{Cu}_{4} \mathrm{I}_{4}$ in solvent.

\begin{tabular}{|c|c|c|c|c|c|}
\hline Solvent & $\begin{array}{c}\text { (R-MBA })_{4} \mathrm{Cu}_{4} \mathrm{I}_{4} \text { in } \\
\text { solvent under } 365 \\
\text { nm irradiation }\end{array}$ & Results & Solvent & $\begin{array}{c}(\mathrm{R}-\mathrm{MBA})_{4} \mathrm{Cu}_{4} \mathrm{I}_{4} \text { in } \\
\text { solvent under } 365 \mathrm{~nm} \\
\text { irradiation }\end{array}$ & Results \\
\hline Chloroform & & No PL & $\begin{array}{c}\text { Ethyl acet } \\
\text { oacetate }\end{array}$ & 12 & No PL \\
\hline $\begin{array}{c}\text { Benzyl Alc } \\
\text { ohol }\end{array}$ & & No PL & $\begin{array}{l}\text { Anhydrou } \\
\text { s ether }\end{array}$ & & Insoluble \\
\hline Octane & & Insoluble & $\begin{array}{l}\text { Tetrahydr } \\
\text { ofuran }\end{array}$ & & No PL \\
\hline $\begin{array}{l}\text { Methylene } \\
\text { dichloride }\end{array}$ & & No PL & Acetone & & Insoluble \\
\hline $\begin{array}{l}\text { Chlorobenz } \\
\text { ene }\end{array}$ & & No PL & $\begin{array}{l}\text { Methyl me } \\
\text { thacrylate }\end{array}$ & & No PL \\
\hline Acetonitrile & & No PL & $\begin{array}{c}\text { Isopropan } \\
\text { ol }\end{array}$ & & Insoluble \\
\hline $\begin{array}{l}\text { Dimethyl su } \\
\text { lfoxide }\end{array}$ & 125 & No PL & $\begin{array}{l}\text { Ethanol } \\
\text { amine }\end{array}$ & & No PL \\
\hline
\end{tabular}


(a)

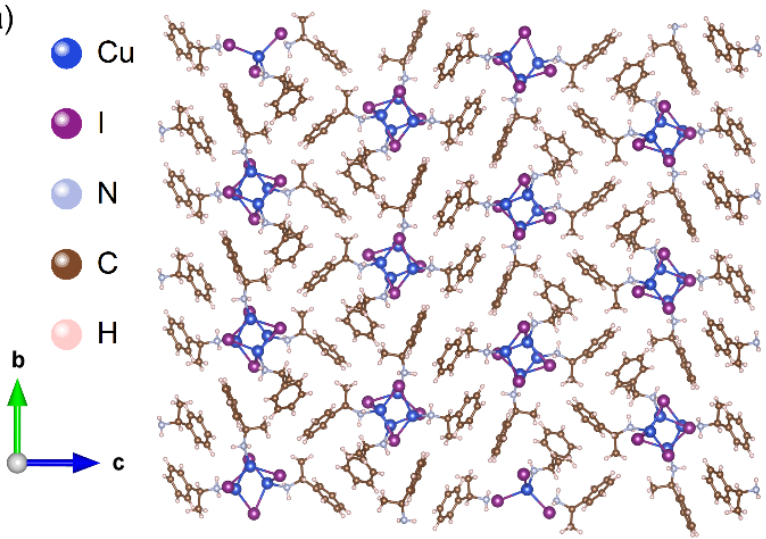

(b)

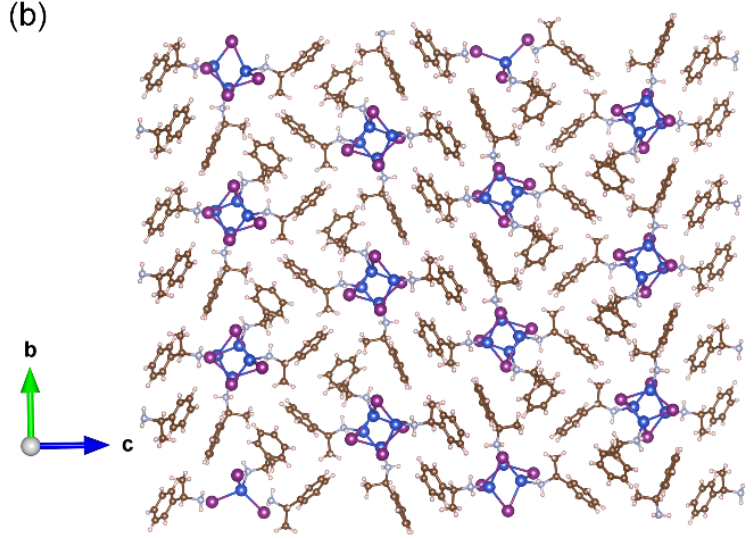

Figure S1. Crystalline structure of (a) (R-MBA) ${ }_{4} \mathrm{Cu}_{4} \mathrm{I}_{4}$ and (b) (S-MBA) ${ }_{4} \mathrm{Cu}_{4} \mathrm{I}_{4}$.

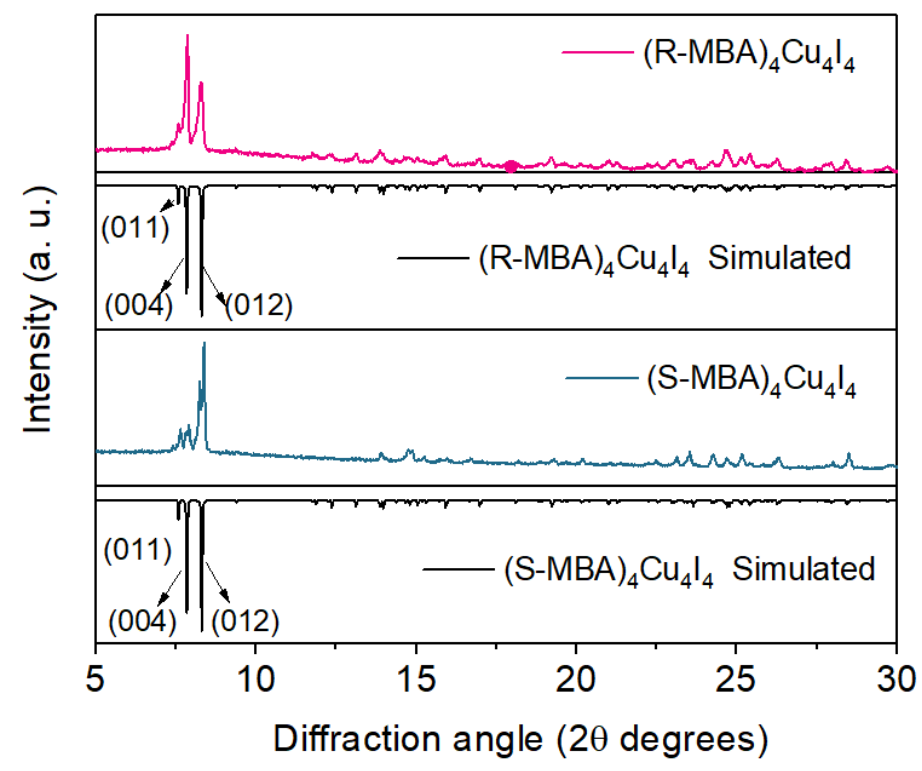

Figure S2. Experimental and calculated powder XRD patterns of $(\mathrm{R}-\mathrm{MBA})_{4} \mathrm{Cu}_{4} \mathrm{I}_{4}$ and $(\mathrm{S}-\mathrm{MBA})_{4} \mathrm{Cu}_{4} \mathrm{I}_{4}$. 


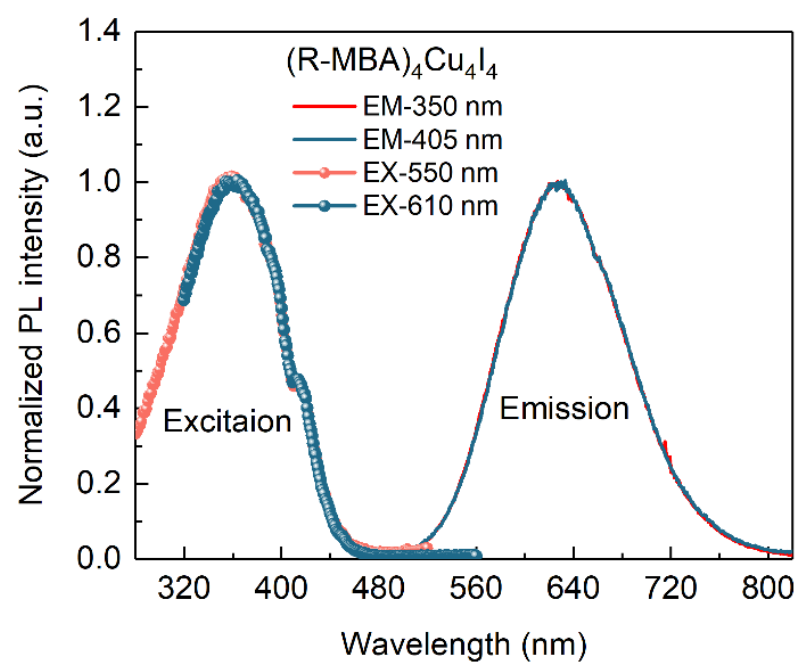

Figure S3. Wavelength dependent photoluminescence excitation and emission spectra of (R-MBA) ${ }_{4} \mathrm{Cu}_{4} \mathrm{I}_{4}$ crystals.

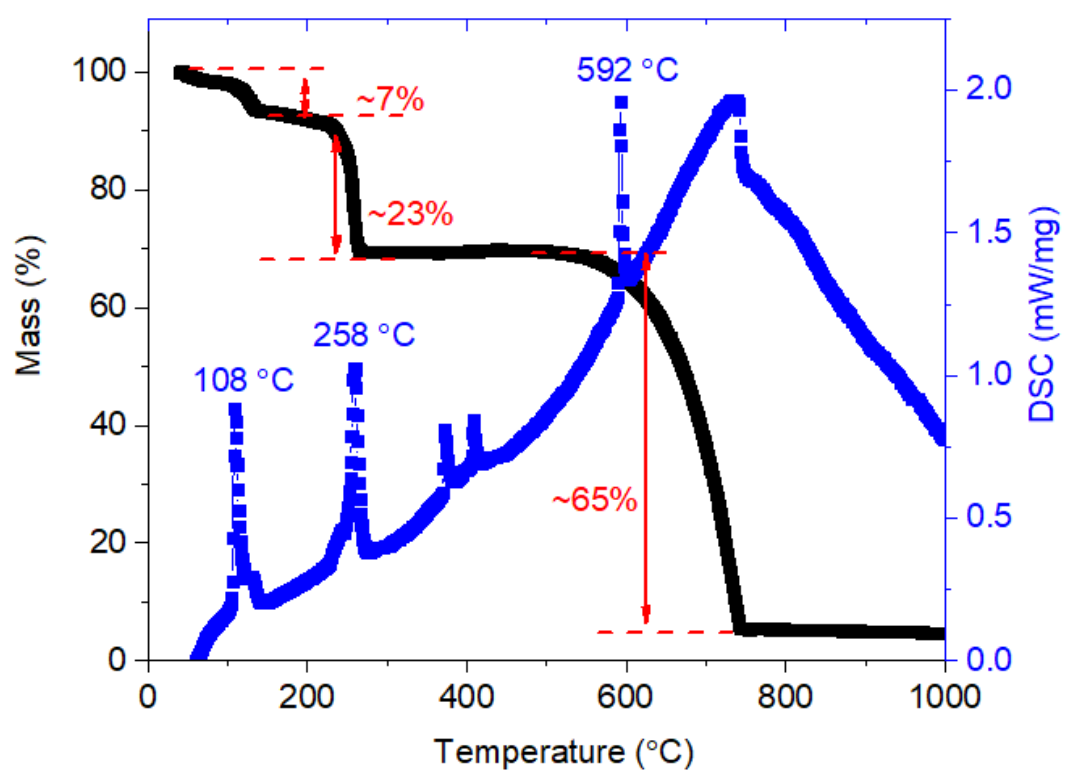

Figure S4. The thermogravimetric analysis (TG) and differential scanning calorimetry (DSC) spectra of (R-MBA) ${ }_{4} \mathrm{Cu}_{4} \mathrm{I}_{4}$. 


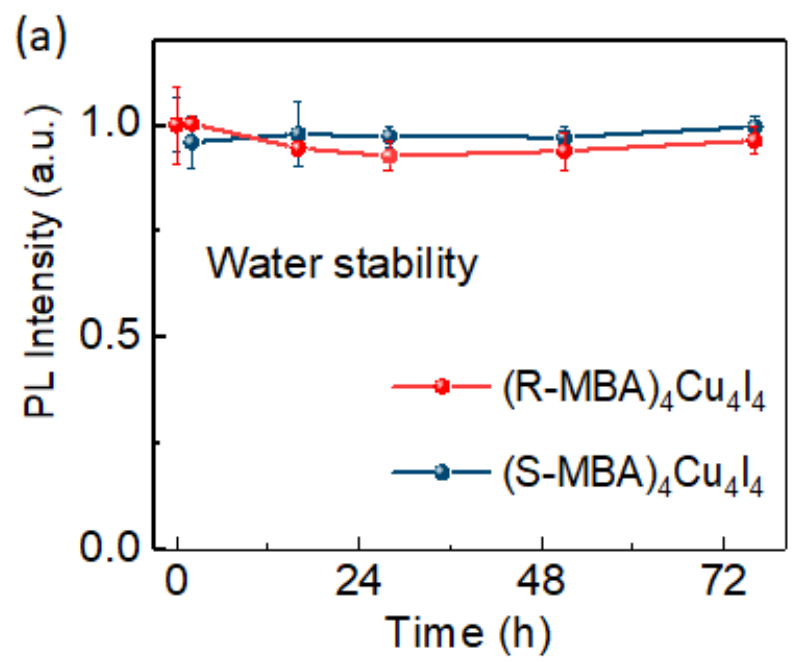

(b)

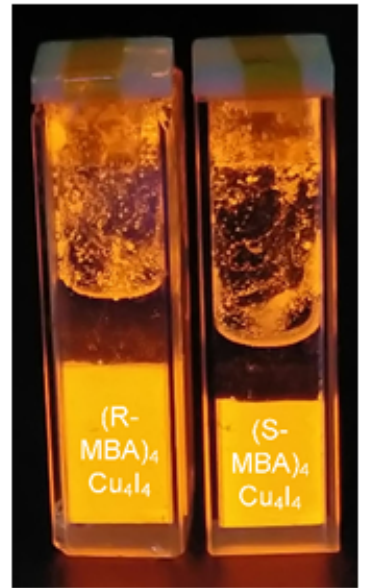

Figure S5. (a) The recorded stability of (R-MBA) ${ }_{4} \mathrm{Cu}_{4} \mathrm{I}_{4}$ and (S-MBA) ${ }_{4} \mathrm{Cu}_{4} \mathrm{I}_{4}$ by soaking in deionized water for 3 days. (b) The photographs of (R-MBA) ${ }_{4} \mathrm{Cu}_{4} \mathrm{I}_{4}$ and (S-MBA) $)_{4} \mathrm{Cu}_{4} \mathrm{I}_{4}$ powders in deionized water under $365 \mathrm{~nm}$ light illumination.

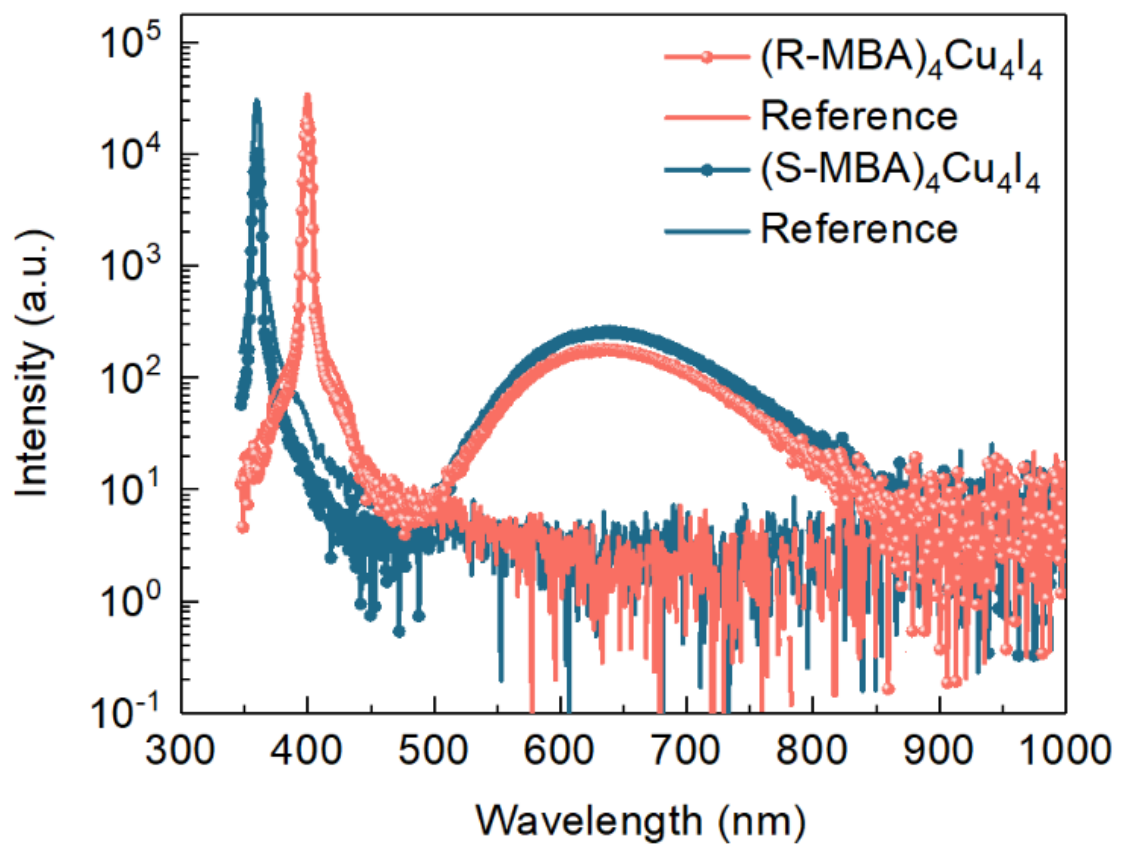

Figure S6. The photoluminescence spectra and reference excitation spectra of (R-MBA) ${ }_{4} \mathrm{Cu}_{4} \mathrm{I}_{4}$ and (S-MBA) ${ }_{4} \mathrm{Cu}_{4} \mathrm{I}_{4}$ clusters collected by an integrating sphere, from which the PLQY of the chiral Cu-I clusters were gotten. 

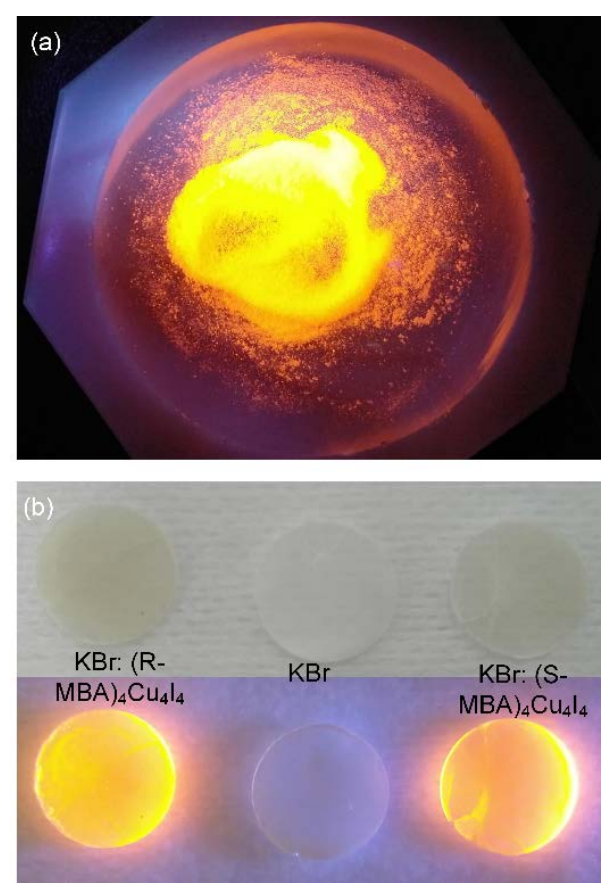

Figure S7. (a) The fine powders prepared by grinding (R-MBA) ${ }_{4} \mathrm{Cu}_{4} \mathrm{I}_{4}$ single crystals with $\mathrm{KBr}$ and the image was taken under $365 \mathrm{~nm}$ irradiation. (b) The pressed pellets containing chiral Cu-I clusters are translucent under ambient light and show strong orange luminescence under $365 \mathrm{~nm}$ irradiation. The KBr pellets are also provided as reference.

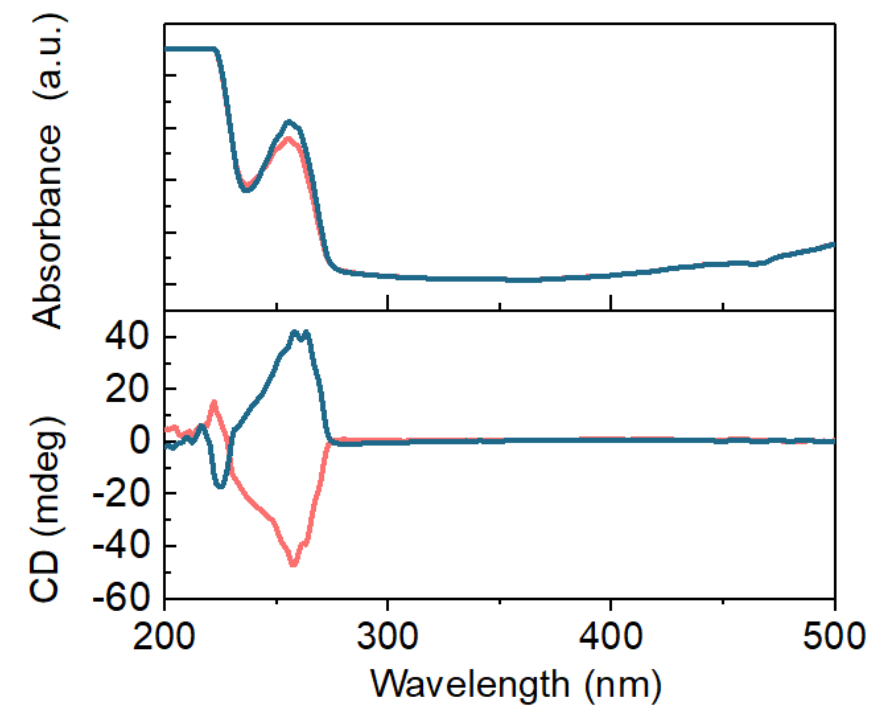

Figure S8. The absorbance spectra and the corresponding CD spectra for the R-MBA and S-MBA organic molecules (dilute with volume ratio of 100:1 in ethanol). 


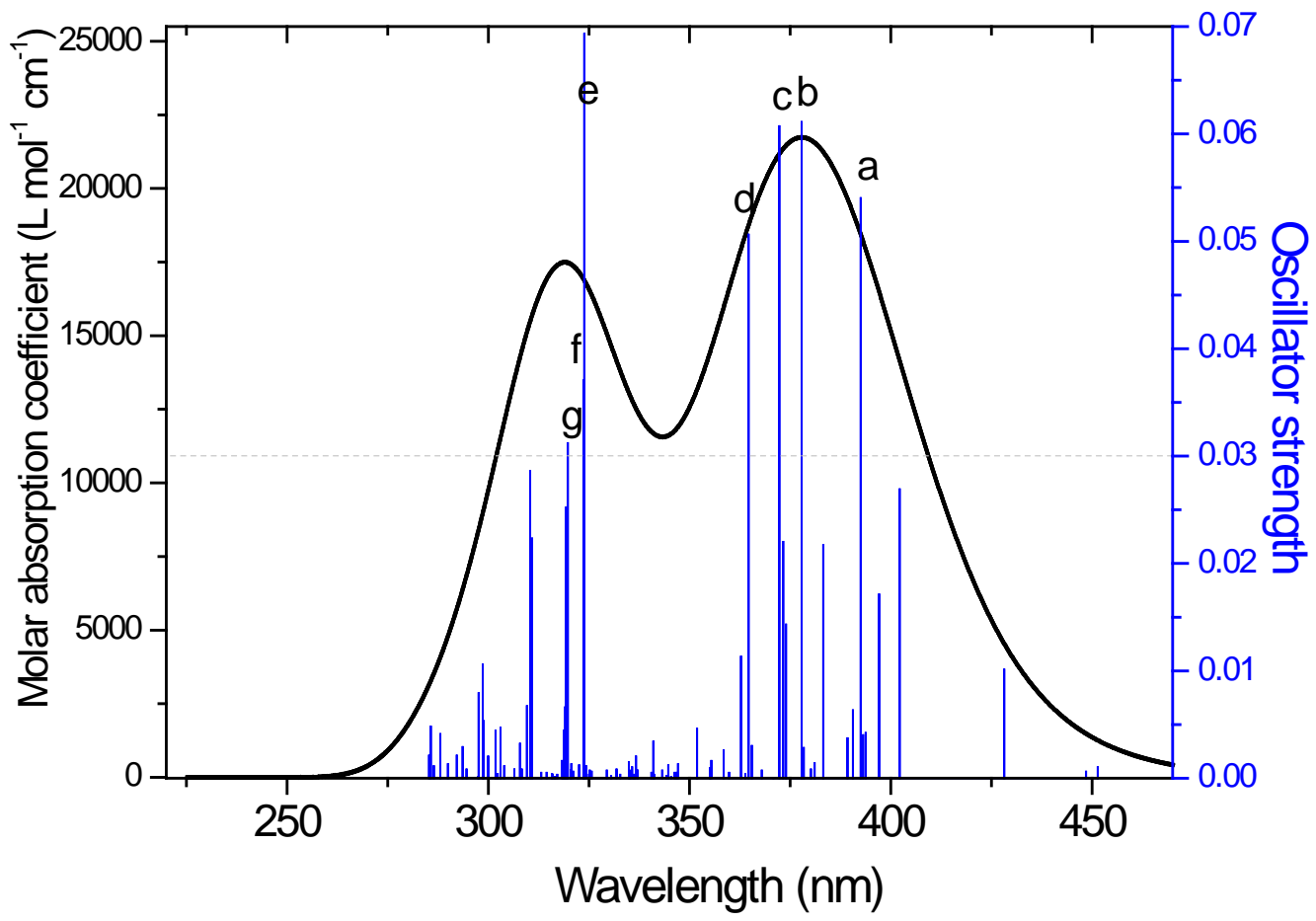

Figure S9. The calculated absorption spectra and oscillator strength of (R-MBA) ${ }_{4} \mathrm{Cu}_{4} \mathrm{I}_{4}$ (number of states=100). 

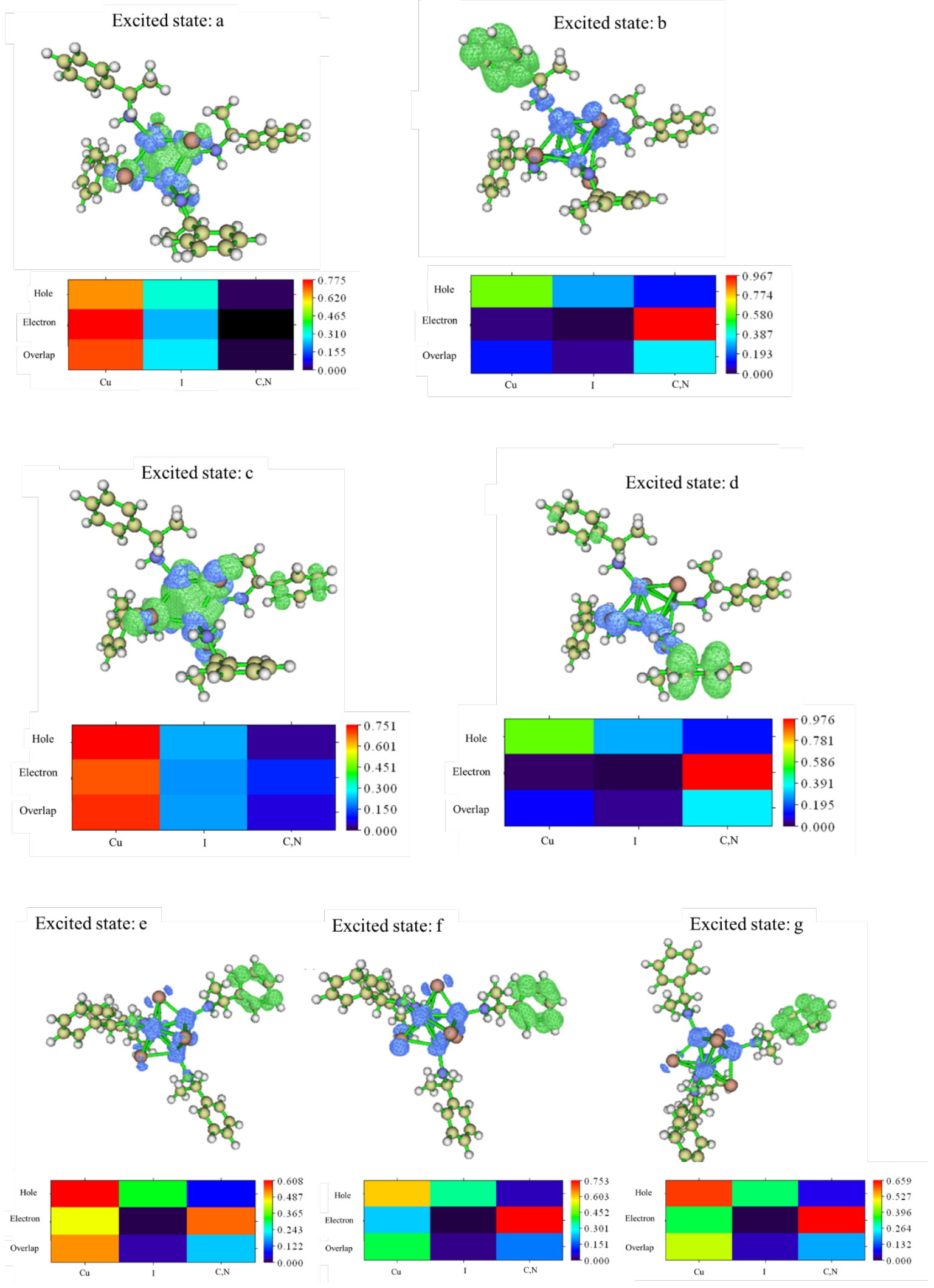

Figure S10. The hole-electron distributions for seven mainly excited states (noted as excited states a, b, c, d, e, f, g, with oscillator strength larger than 0.03 ) belong to two absorption peak seperately. The hole and electron distribution are shown as blue and green isosurfaces in the 
cluster structure, respectively. The hole/electron composition and overlap was also plotted as heatmap just below. The color in the heatmap represent the contribution of each fragment $(\mathrm{Cu}, \mathrm{I}$ and ligand (containing $\mathrm{C}, \mathrm{N}$ )) to hole and electron. Generally, the charges transfer from the hole to electron sites.

\section{References}

1. Ahn, J.; Lee, E.; Tan, J.; Yang, W.; Kim, B.; Moon, J. A new class of chiral semiconductors: chiral-organic-molecule-incorporating organic-inorganic hybrid perovskites. Mater. Horiz. 2017, 4, 851-856.

2. Kresse, G.; Furthmüller, J. Efficient iterative schemes for ab initio total-energy calculations using a plane-wave basis set. Phys. Rev. B 1996, 54, 11169-11186.

3. Perdew, J. P.; Burke, K.; Ernzerhof, M. Generalized Gradient Approximation Made Simple. Phys. Rev. Lett. 1996, 77, 3865-3868.

4. Monkhorst, H. J.; Pack, J. D. Special points for Brillouin-zone integrations. Phys. Rev. B 1976, 13, 5188-5192.

5. M. J. Frisch, G. W. T., H. B. Schlegel, G. E. Scuseria, M. A. Robb, J. R. Cheeseman, G. Scalmani, V. Barone, G. A. Petersson, H. Nakatsuji, X. Li, M. Caricato, A. V. Marenich, J. Bloino, B. G. Janesko, R. Gomperts, B. Mennucci, H. P. Hratchian, J. V. Ortiz, A. F. Izmaylov, J. L. Sonnenberg, D. Williams-Young, F. Ding, F. Lipparini, F. Egidi, J. Goings, B. Peng, A. Petrone, T. Henderson, D. Ranasinghe, V. G. Zakrzewski, J. Gao, N. Rega, G. Zheng, W. Liang, M. Hada, M. Ehara, K. Toyota, R. Fukuda, J. Hasegawa, M. Ishida, T. Nakajima, Y. Honda, O. Kitao, H. Nakai, T. Vreven, K. Throssell, J. A. Montgomery, Jr., J. E. Peralta, F. Ogliaro, M. J. Bearpark, J. J. Heyd, E. N. Brothers, K. N. Kudin, V. N. Staroverov, T. A. Keith, R. Kobayashi, J. Normand, ; K. Raghavachari, A. P. R., J. C. Burant, S. S. Iyengar, J. Tomasi, M. Cossi, J. M. Millam, M. Klene, C. Adamo, R. Cammi, J. W. Ochterski, R. L. Martin, K. Morokuma, O. Farkas, J. B. Foresman, and D. J. Fox, , Gaussian 16, Revision A.03,. Gaussian, Inc., 2016, Wallingford CT, 2016.

6. Perdew, J. P.; Burke, K.; Ernzerhof, M., Perdew, Burke, and Ernzerhof Reply. Phys. Rev. Lett. 1998, 80, 891-891.

7. Hirshfeld, F. L., Bonded-atom fragments for describing molecular charge densities. Theoretica chimica acta 1977, 44, 129-138.

8. Lu, T.; Chen, F. Multiwfn: A multifunctional wavefunction analyzer. J. Comput. Chem. 2012, 33, 580-592.

9. Zinna, F.; Giovanella, U.; Di Bari, L. Highly circularly polarized electroluminescence from a chiral europium complex. Adv. Mater. 2015, 27, 1791-5.

10. Bozoklu, G.; Gateau, C.; Imbert, D.; Pecaut, J.; Robeyns, K.; Filinchuk, Y.; Memon, F.; Muller, G.; Mazzanti, M. Metal-controlled diastereoselective self-assembly and circularly polarized luminescence of a chiral heptanuclear europium wheel. J. Am. Chem. Soc. 2012, 134, 8372-5.

11. Petoud, S.; Muller, G.; Moore, E. G.; Xu, J.; Sokolnicki, J.; Riehl, J. P.; Le, U. N.; Cohen, S. M.; Raymond, K. N. Brilliant Sm, Eu, Tb, and Dy Chiral Lanthanide Complexes with Strong Circularly Polarized Luminescence. J. Am. Chem. Soc. 2007, 129, 77-83.

12. Kawai, T.; Kawamura, K.; Tsumatori, H.; Ishikawa, M.; Naito, M.; Fujiki, M.; Nakashima, T. 
Circularly polarized luminescence of a fluorescent chiral binaphtylene-perylenebiscarboxydiimide dimer. Chemphyschem 2007, 8, 1465-8.

13. Sawada, Y.; Furumi, S.; Takai, A.; Takeuchi, M.; Noguchi, K.; Tanaka, K. Rhodium-catalyzed enantioselective synthesis, crystal structures, and photophysical properties of helically chiral 1,1'-bitriphenylenes. J. Am. Chem. Soc. 2012, 134, 4080-3.

14. Takaishi, K.; Yamamoto, T.; Hinoide, S.; Ema, T. Helical Oligonaphthodioxepins Showing Intense Circularly Polarized Luminescence (CPL) in Solution and in the Solid State. Chem.Euro. J.l 2017, 23, 9249-9252.

15. Jiang, X.; Liu, X.; Jiang, Y.; Quan, Y.; Cheng, Y.; Zhu, C. Fluorescence Study of Chiral $\beta$-Ketoiminate-Based Newly Synthesized Boron Hybrid Polymers. Macromol. Chem. Phys. 2014, 215, 358-364.

16. Schaffner-Hamann, C.; von Zelewsky, A.; Barbieri, A.; Barigelletti, F.; Muller, G.; Riehl, J. P.; Neels, A. Diastereoselective Formation of Chiral Tris-Cyclometalated Iridium (III) Complexes: Characterization and Photophysical Properties. J. Am. Chem. Soc. 2004, 126, 9339-9348.

17. Kogel, J. F.; Kusaka, S.; Sakamoto, R.; Iwashima, T.; Tsuchiya, M.; Toyoda, R.; Matsuoka, R.; Tsukamoto, T.; Yuasa, J.; Kitagawa, Y.; Kawai, T.; Nishihara, H. Heteroleptic [Bis(oxazoline)](dipyrrinato)zinc(II) Complexes: Bright and Circularly Polarized Luminescence from an Originally Achiral Dipyrrinato Ligand. Angew. Chem. 2016, 55, $1377-81$.

18. Shi, L.; Zhu, L.; Guo, J.; Zhang, L.; Shi, Y.; Zhang, Y.; Hou, K.; Zheng, Y.; Zhu, Y.; Lv, J.; Liu, S.; Tang, Z. Self-Assembly of Chiral Gold Clusters into Crystalline Nanocubes of Exceptional Optical Activity. Angew. Chem. 2017, 56, 15397-15401.

19. Jin, Y.; Li, S.; Han, Z.; Yan, B. J.; Li, H. Y.; Dong, X. Y.; Zang, S. Q. Cations Controlling the Chiral Assembly of Luminescent Atomically Precise Copper(I) Clusters. Angew. Chem. 2019, 58, 12143-12148.

20. Long, G.; Jiang, C.; Sabatini, R.; Yang, Z.; Wei, M.; Quan, L. N.; Liang, Q.; Rasmita, A.; Askerka, M.; Walters, G.; Gong, X.; Xing, J.; Wen, X.; Quintero-Bermudez, R.; Yuan, H.; Xing, G.; Wang, X. R.; Song, D.; Voznyy, O.; Zhang, M.; Hoogland, S.; Gao, W.; Xiong, Q.; Sargent, E. H. Spin control in reduced-dimensional chiral perovskites. Nat. Photonics 2018, 12, 528-533.

21. Ma, J.; Fang, C.; Chen, C.; Jin, L.; Wang, J.; Wang, S.; Tang, J.; Li, D. Chiral 2D Perovskites with a High Degree of Circularly Polarized Photoluminescence. ACS Nano 2019, 13, 3659-3665.

22. Wang, J.; Fang, C.; Ma, J.; Wang, S.; Jin, L.; Li, W.; Li, D. Aqueous Synthesis of Low-Dimensional Lead Halide Perovskites for Room-Temperature Circularly Polarized Light Emission and Detection. ACS Nano 2019, 13, 9473-9481.

23. Chen, W.; Zhang, S.; Zhou, M.; Zhao, T.; Qin, X.; Liu, X.; Liu, M.; Duan, P. Two-Photon Absorption-Based Upconverted Circularly Polarized Luminescence Generated in Chiral Perovskite Nanocrystals. J. Phys. Chem. Lett. 2019, 10, 3290-3295.

24. Shi, Y.; Duan, P.; Huo, S.; Li, Y.; Liu, M. Endowing Perovskite Nanocrystals with Circularly Polarized Luminescence. Adv. Mater. 2018, 30, e1705011. 